\title{
$\begin{array}{llll}\text { J.Thi-Qar Sci. } & \text { No. (2) Vol.1 Aug./2008 }\end{array}$
}

ISSN 1991- 8690

website: http://jsci.utq.edu.iq

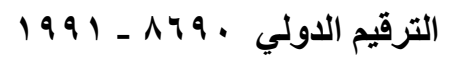

Email: utjsci@utq.edu.iq

Some types of fuzzy ideals in semigroups

\author{
Rabee Hadi Jari Akram Barazan Attar \\ Department of Mathematics - College of Education \\ University of Thi-Qar.
}

\begin{abstract}
$\underline{\text { Abstract }}$
In this paper,we study the notion of fuzzy ideal in semigroups and give some properties about it and we reviewed some types of ideals such as (regular,semiprime,(1,2)- ideal,(2,2)-ideal and gives some relationships between them.
\end{abstract}




\section{J.Thi-Qar Sci.}

\section{Introduction:}

As a continuation to the study of fuzzy sets which initiated in[1] and which have been studied by Goguen in [2] and several researchers explored on the generalization of the notion of fuzzy set.The concept of an intuitionistic fuzzy set was introduced byAtanssov K.Y.[3], as a generalization of the notion of fuzzy set.Fuzzy ideals and fuzzy biideal,(1,2)-ideals in semigroup was introduced by S.Lajos and N.Kuroki [4,5].Our interest in this paper is to study some of their important properties.

\section{Preliminaries:}

In this section, we shall give the concepts of fuzzy sets and basic definitions with some related properties which will be used in the next sections.

\section{Definition 2.1[1]:}

A fuzzy set in a set $M$ is a mapping $X$ from a nonempty set $M$ into [0, 1].

\section{Definition 2.2 [1] :}

Let $A$ and $B$ be two fuzzy sets in $M$.Then:

1. $A \subseteq B$ if and only if $A(x) \leq B(x)$

2. $(A \cap B)(x)=\min \{A(x), B(x)\}$, for all $x \in M$.

\section{Definition 2.3[6]:}

A fuzzy set $X$ in a ring $R$ is called a fuzzy ideal of $R$ if for each $x, y \in R$.

$1-X(x-y) \geq \min \{X(x), X(y)\}$.

2- $X(x y) \geq \max \{X(x), X(y)\}$.

\section{Intuitionistic Q- fuzzy ideals}

\section{Definition 3.1[7]:}

An intuitionistic Q-fuzzy set A is an object having the form $A=\left\{\left(x, \mu_{A}(x, q), \gamma_{A}(x, q)\right): x \in X, q \in Q\right\}$ where the function $\mu_{A}: X \times Q \rightarrow[0,1]$ and $\gamma_{A}: X \times Q \rightarrow[0,1]$ denoted by the degree of membership(namely $\left.\mu_{A}(x, q)\right)$ and the degree of nonmembership $\gamma_{A}(x, q)$ of each element $(x, q) \in X \times Q$ to the set $\mathrm{A}$, respectively, and $0 \leq \mu_{A}(x, q)+\gamma_{A}(x, q) \leq 1$ for all $x \in X$ and $q \in Q$. 


\section{$\begin{array}{llll}\text { J.Thi-Qar Sci. } & \text { No. (2) } & \text { Vol.1 } & \text { Aug./2008 }\end{array}$}

For the sake of simplicity, we shall use the symbol $A=\left(\mu_{A}, \gamma_{A}\right)$ for the IQFS $A=\left\{\left(x, \mu_{A}(x, q), \gamma_{A}(x, q)\right): x \in x, q \in Q\right\}$.

\section{Definition 3.2 [7] :}

An IQFS $A=\left(\mu_{A}, \gamma_{A}\right)$ in $\mathrm{S}$ is called an intuitionistic Q-fuzzy subsemigroup of $\mathrm{S}$ if

1. $\mu_{A}(x y, q) \geq \min \left\{\mu_{A}(x, q), \mu_{A}(y, q)\right\}$.

2. $\gamma_{A}(x y, q) \leq \max \left\{\gamma_{A}(x, q), \gamma_{A}(y, q)\right\}$, for all $x, y \in S$ and $\mathrm{q} \in \mathrm{Q}$.

\section{Definition 3.3 [7]:}

An IQFS $A=\left(\mu_{A}, \gamma_{A}\right)$ in $\mathrm{S}$ is called an intuitionistic Q-fuzzy left ideal of $\mathrm{S}$ if

1. $\mu_{A}(x y, q) \geq \mu_{A}(y, q)$,

2. $\gamma_{A}(x y, q) \leq \gamma_{A}(y, q)$, for all $\mathrm{x}, \mathrm{y} \in \mathrm{S}$ and $\mathrm{q} \in \mathrm{Q}$.

\section{Definition 3.4 [7]:}

An intuitionistic Q-fuzzy intuitionistic subsemigroup $A=\left(\mu_{A}, \gamma_{A}\right)$ of $\mathrm{S}$ is called an intuitionistic Q-fuzzy bi-ideal of S if

1. $\mu_{A}(x w y, q) \geq \min \left\{\mu_{A}(x, q), \mu_{A}(y, q)\right\}$,

2. . $\gamma_{A}(x w y, q) \leq \max \left\{\gamma_{A}(x, q), \gamma_{A}(y, q)\right\}$, for all $x, y, w \in S$ and $\mathrm{q} \in \mathrm{Q}$.

\section{Proposition 3.5:}

If $A_{i}$ is an intuitionistic Q-fuzzy bi-ideal of $\mathrm{S} \forall \mathrm{i} \in \wedge$, then $\cap A_{i}$ is an intuitionistic Q-fuzzy bi-ideal , where $\cap A_{i}=\left(\wedge \mu_{A i}, \vee \gamma_{A i}\right)$ and $\wedge \mu_{A i}(x)=\inf \left\{\mu_{A i}(x) / \mathrm{i} \in \wedge, \mathrm{x} \in \mathrm{S}\right\}$, $\vee \gamma_{A_{i}}(x)=\sup \left\{\gamma_{A_{i}}(x) / i \in \wedge, x \in S\right\}$.

\section{Proof:}

To prove $\cap A_{i}$ is an intuitionistic Q-fuzzy subsemigroup

$\wedge \mu_{A_{i}}(x y, q) \geq \wedge\left\{\min \left\{\mu_{A_{i}}(x, q), \mu_{A_{i}}(y, q)\right\}\right\}$

$=\min \left\{\min \left\{\mu_{A_{i}}(x, q), \mu_{A_{i}}(y, q)\right\}\right\}=\min \left\{\min \left\{\mu_{A_{i}}(x, q), \min \mu_{A_{i}}(y, q)\right\}\right\}=$ $\min \left\{\wedge\left\{\mu_{A_{i}}(x, q), \wedge \mu_{A_{i}}(y, q)\right\}\right\}$

and

$$
\begin{aligned}
& \vee \gamma_{A_{i}}(x y, q) \leq \vee\left\{\max \left\{\gamma_{A_{i}}(x, q), \gamma_{A i}(y, q)\right\}\right\}=\max \left\{\max \left\{\gamma_{A_{i}}(x, q), \gamma_{A_{i}}(y, q)\right\}\right\}= \\
& \left\{\max \left\{\gamma_{A_{i}}(x, q), \max \gamma_{A_{i}}(y, q)\right\}\right\}=\max \left\{\vee\left\{\gamma_{A_{i}}(x, q), \vee \gamma_{A_{i}}(y, q)\right\}\right\} .
\end{aligned}
$$




\section{$\begin{array}{llll}\text { J.Thi-Qar Sci. No. (2) } & \text { Vol.1 } & \text { Aug./2008 }\end{array}$}

Thus, $\cap A_{i}$ is an intuitionistic Q-fuzzy intuitionistic subsemigroup of $\mathrm{S}$.

To prove $\cap A_{i}$ is Q-fuzzy bi-ideal of $\mathrm{S}$.

Let $x, y, a \in S$, then $\mu_{A_{i}}(x a y, q) \geq \wedge\left\{\min \left\{\mu_{A_{i}}(x, q), \mu_{A_{i}}(y, q)\right\}\right\}=$

$\min \left\{\min \left\{\mu_{A_{i}}(x, q), \mu_{A_{i}}(y, q)\right\}\right\}=\min \left\{\min \left\{\mu_{A_{i}}(x, q), \min \mu_{A_{i}}(y, q)\right\}\right\}=$

$\min \left\{\wedge\left\{\mu_{A_{i}}(x, q), \wedge \mu_{A_{i}}(y, q)\right\}\right\}$

and

$\vee \gamma_{A_{i}}(x a y, q) \leq \vee\left\{\max \left\{\gamma_{A_{i}}(x, q), \gamma_{A_{i}}(y, q)\right\}\right\}=\max \left\{\max \left\{\gamma_{A_{i}}(x, q), \gamma_{A_{i}}(y, q)\right\}\right\}=$

$\max \left\{\max \left\{\gamma_{A_{i}}(x, q), \max \gamma_{A_{i}}(y, q)\right\}\right\}=\max \left\{\vee \gamma_{A_{i}}(x, q), \vee \gamma_{A_{i}}(y, q)\right\}$.

Hence, $\cap A_{i}$ is intuitionistic Q-fuzzy bi-ideal of $\mathrm{S}$.

\section{Definition 3.6 [8]:}

An intuitionistic Q-fuzzy intuitionistic subsemigroup $A=\left(\mu_{A}, \gamma_{A}\right)$ of $\mathrm{S}$ is called an intuitionistic fuzzy $(1,2)$-ideal of $\mathrm{S}$ if

1. $\mu_{A}(x w(y z), q) \geq \min \left\{\mu_{A}(x, q), \mu_{A}(y, q), \mu_{A}(z, q)\right\}$,

2. $\gamma_{A}(x w(y z), q) \leq \max \left\{\gamma_{A}(x, q), \gamma_{A}(y, q), \gamma_{A}(z, q)\right\}$ for all $\mathrm{w}, \mathrm{x}, \mathrm{y}, \mathrm{z} \in \mathrm{S}$ and $\mathrm{q} \in \mathrm{Q}$.

\section{Proposition 3.7:}

Every intuitionistic Q-fuzzy bi-ideal is an intuitionistic Q-fuzzy (1,2)-ideal.

\section{Proof:}

Let $A=\left(\mu_{A}, \gamma_{A}\right)$ be an intuitionistic Q-fuzzy intuitionistic bi-ideal of $\mathrm{S}$ and let $w, x, y, z \in S$ and $q \in Q$,then

$\mu_{A}(x w(y z), q)=\mu_{A}(x(w y) z, q) \geq \min \left\{\mu_{A}((x w y), q), \mu_{A}(z, q)\right\} \geq$

$\min \left\{\min \left\{\mu_{A}(x, q), \mu_{A}(y, q), \mu_{A}(z, q)\right\}\right\}=\min \left\{\left\{\mu_{A}(x, q), \mu_{A}(y, q), \mu_{A}(z, q)\right\}\right\}$

and

$\left.\gamma_{A}(x w(y z), q)=\gamma_{A}(x w y) z, q\right) \leq \max \left\{\gamma_{A}((x w y), q), \gamma_{A}(z, q)\right\}=$

$\max \left\{\gamma_{A}(x, q), \gamma_{A}(y, q), \gamma_{A}(z, q)\right\}$.

Thus, $A=\left(\mu_{A}, \gamma_{A}\right)$ is an intuitionistic Q-fuzzy (1,2)-ideal of S.

Recall that a semigroup $\mathrm{S}$ is said to be regular if, for each $x \in S$, there exists $y \in S$ such that $\mathrm{x}=\mathrm{xyx},[9]$. 


\section{$\begin{array}{llll}\text { J.Thi-Qar Sci. } & \text { No. (2) Vol.1 } & \text { Aug./2008 }\end{array}$}

\section{Proposition 3.8:}

If $S$ is a regular semigroup,then every intuitionistic Q-fuzzy $(1,2)$-ideal of $S$ is an intuitionistic Q-fuzzy bi-ideal.

\section{Proof:}

Suppose that $\mathrm{S}$ is a regular semigroup and let $A=\left(\mu_{A}, \gamma_{A}\right)$ be an intuitionistic Q-fuzzy (1,2)-ideal of $\mathrm{S}$.

Let $w, x, y \in S$, since $S$ regular, then $x w \in(x S x) S \subseteq x S$, it follows $\mathrm{xw}=\mathrm{xsx}$ for some $s \in S$,consequently $\mu_{A}(x w y, q)=\mu_{A}((x s x) y, q)=\mu_{A}(x s(x y), q) \geq \min \left\{\mu_{A}(x, q), \mu_{A}(x, q), \mu_{A}(y, q)\right\}=$ $\min \left\{\mu_{A}(x, q), \mu_{A}(y, q)\right\}$

and $\gamma_{A}((x w y), q)=\gamma_{A}((x s x) y, q)=\gamma_{A}(x s(x y), q) \leq \max \left\{\gamma_{A}(x, q), \gamma_{A}(x, q), \gamma_{A}(y, q)\right\}=$ $\max \left\{\gamma_{A}(x, q), \gamma_{A}(y, q)\right\}$

Hence $A=\left(\mu_{A}, \gamma_{A}\right)$ is intuitionistic Q-fuzzy bi-ideal of S.

Recall that, A semigroup $\mathrm{S}$ is said to be (2,2)-regular if $x \in x^{2} S x^{2}$ for all $x \in S,[9,10]$.

\section{Proposition 3.9:}

Let $A=\left(\mu_{A}, \gamma_{A}\right)$ be an intuitionistic Q-fuzzy bi-ideal of S.If S is a (2,2)-regular, then $A(a, q)=A\left(a^{2}, q\right)$ for all $a \in S$.

\section{Proof:}

Let $a \in S$, since $S$ is (2,2)-regular, then $a \in a^{2} S a^{2}$,implies that $a=a^{2} x a^{2}$ for some $x \in S$.

Now, $\mu_{A}(a, q)=\mu_{A}\left(a^{2} x a^{2}, q\right) \geq \min \left\{\mu_{A}\left(a^{2}, q\right), \mu_{A}\left(a^{2}, q\right)\right\}=\mu_{A}\left(a^{2}, q\right)$

$\geq \min \left\{\mu_{A}(a, q), \mu_{A}(a, q)\right\}=\mu_{A}(a, q)$

also,

$\gamma_{A}(a, q)=\gamma_{A}\left(a^{2} x a^{2}, q\right) \leq \max \left\{\gamma_{A}\left(a^{2}, q\right), \gamma_{A}\left(a^{2}, q\right)\right\}=\gamma_{A}\left(a^{2}, q\right) \leq \max \left\{\gamma_{A}(a, q), \gamma_{A}(a, q)\right\}=$ $\gamma_{A}(a, q)$.

Hence, $\mu_{A}(a, q)=\mu_{A}\left(a^{2}, q\right)$ and $\gamma_{A}(a, q)=\gamma_{A}\left(a^{2}, q\right)$; that is $A(a, q)=A\left(a^{2}, q\right)$. 


\section{$\begin{array}{llll}\text { J.Thi-Qar Sci. No. (2) } & \text { Vol.1 } & \text { Aug./2008 }\end{array}$}

Recall that, a semigroup $\mathrm{S}$ is called intra-regular if, for each element a of $\mathrm{S}$, there exists elements $\mathrm{x}$ and $\mathrm{y}$ in $\mathrm{S}$ such that $a=x a^{2} y,[9]$.

\section{Proposition 3.10:}

Let $A=\left(\mu_{A}, \gamma_{A}\right)$ be an intuitionistic Q-fuzzy ideal of $\mathrm{S}$.If $\mathrm{S}$ is an intra-regular, then $A(a, q)=A\left(a^{2}, q\right)$ for all $a \in S$ and $q \in Q$.

\section{Proof:}

L et $a \in S$, since $S$ is intra-regular, there exist $\mathrm{x}$ and $\mathrm{y} \in S$ such that $a=x a^{2} y$.

Now, $\mu_{A}(a, q)=\mu_{A}\left(x a^{2} y, q\right) \geq \mu_{A}\left(x a^{2}, q\right) \geq \mu_{A}\left(a^{2}, q\right) \geq\left\{\mu_{A}(a, q), \mu_{A}(a, q)\right\}=\mu_{A}(a, q)$.

Also, $\gamma_{A}(a, q)=\gamma_{A}\left(x a^{2} y, q\right)=\gamma_{A}\left(x a^{2}, q\right) \leq \gamma_{A}\left(a^{2}, q\right) \leq \max \left\{\gamma_{A}(a, q), \gamma_{A}(a, q)\right\}=\gamma_{A}(a, q)$.

Since $\mu_{A}(a, q)=\mu_{A}\left(a^{2}, q\right)$ and $\gamma_{A}(a, q)=\gamma_{A}\left(a^{2}, q\right)$, then $A(a, q)=A\left(a^{2}, q\right)$.

\section{Corollary3.11 ;}

Let $A=\left(\mu_{A}, \gamma_{A}\right)$ be an intuitionistic Q-fuzzy ideal of $\mathrm{S}$.If $\mathrm{S}$ is intra-regular, then $A(a b, q)=A(b a, q)$ for all $a, b \in S$.

\section{Proof:}

Let $a, b \in S$. Then $\quad$ Py $\quad 3.10$,

$\mu_{A}(a b, q)=\mu_{A}\left((a b)^{2}, q\right) \geq \mu_{A}\left((a(b a) b, q) \geq \mu_{A}(b a, q)=\mu_{A}\left((b a)^{2}, q\right)\right.$

$\geq \mu_{A}(b(a b) a, q) \geq \mu_{A}(a b, q)$.

and

$\gamma_{A}(a b, q)=\gamma_{A}\left((a b)^{2}, q\right)=\gamma_{A}(a(b a) b, q) \leq \gamma_{A}(b a, q)=\gamma_{A}\left((b a)^{2}, q\right)=$ $\gamma_{A}(b(a b) a, q) \leq \gamma_{A}(a b)$.

Hence $\mu_{A}(a b, q)=\mu_{A}(b a, q)$ and $\gamma_{A}(a b, q)=\gamma_{A}(b a, q)$, so $A(a b, q)=A(b a, q)$.

\section{Intuitionistic fuzzy ideals}

\section{Definition 4.1[10]:}

An intuitionistic fuzzy set (brifly,IFS) $\mathrm{A}$ in a non-empty set $\mathrm{X}$ is an object having the form $A=\left\{\left(x, \mu_{a}(x), \gamma_{A}(x)\right): x \in X\right\}$ where the function $\mu_{A}: X \rightarrow[0,1]$ and $\gamma_{A}: X \rightarrow[0,1]$ denoted by the degree of membership and the degree of non 


\section{J.Thi-Qar Sci. No. (2) Vol.1 Aug./2008}

membership,respectively, and $0 \leq \mu_{A}(x)+\gamma_{A}(x) \leq 1$ for all $x \in X$.An intuitionistic fuzzy

set $A=\left\{\left(x, \mu_{A}(x), \gamma_{A}(x)\right): x \in X\right\}$ in $X$ can be identified to an order pair $\left(\mu_{A}, \gamma_{A}\right)$ in $I^{X} \times I^{X}$.For the sake of simplicity, denoted by the symbol $A=\left(\mu_{A}, \gamma_{A}\right)$ for the IFS $A=\left\{\left(x, \mu_{A}(x), \gamma_{A}(x)\right): x \in X\right\}$.

\section{Definition 4.2 [9]:}

An IFS $A=\left(\mu_{A}, \gamma_{A}\right)$ in $S$ is called an intuitionistic fuzzy subsemigroup of $\mathrm{S}$ if

1. $\mu_{A}(x y) \geq \min \left\{\mu_{A}(x), \mu_{A}(y)\right\}$.

2. $\gamma_{A}(x y) \leq \max \left\{\gamma_{A}(x), \gamma_{A}(y)\right\}$, for all $x, y \in S$.

\section{Definition 4.3 [9]:}

An IFS $A=\left(\mu_{A}, \gamma_{A}\right)$ in $\mathrm{S}$ is called an intuitionistic fuzzy left ideal of $\mathrm{S}$ if

1. $\mu_{A}(x y) \geq \mu_{A}(y)$,

2. $\gamma_{A}(x y) \leq \gamma_{A}(y)$, for all $\mathrm{x}, \mathrm{y} \in \mathrm{S}$.

\section{Definition 4.4 [3]:}

An IFS $A=\left(\mu_{A}, \gamma_{A}\right)$ in $\mathrm{S}$ is called intuitionistic fuzzy semiprime if

1. $\mu_{A}(x) \geq \mu_{A}\left(x^{2}\right)$

2. $\gamma_{A}(x) \leq \gamma_{A}\left(x^{2}\right)$, for all $x \in S$.

\section{Proposition 4.5 :}

For any intuitionistic fuzzy subsemigroup $A=\left(\mu_{A}, \gamma_{A}\right)$ of $\mathrm{S}$, if $A=\left(\mu_{A}, \gamma_{A}\right)$ is intuitionistic fuzzy semiprime, then $A(a)=A\left(a^{2}\right)$.

\section{Proof:}

Let $a \in S$.Then, $\mu_{A}(a) \geq \mu_{A}\left(a^{2}\right)=\min \left\{\mu_{A}(a), \mu_{A}(a)\right\}=\mu_{A}(a)$.

So, $\mu_{A}(a)=\mu_{A}\left(a^{2}\right)$

And $\gamma_{a}(a) \leq \gamma_{A}\left(a^{2}\right)=\max \left\{\gamma_{A}(a), \gamma_{A}(a)\right\}=\gamma_{A}(a)$.

Hence, $\gamma_{A}(a)=\gamma_{A}\left(a^{2}\right)$,it follows that $A(a)=A\left(a^{2}\right)$.

Recall that a semigroup is called left regular if for each element a of S,there exists an element $\mathrm{x}$ in $\mathrm{S}$ such that $a=x a^{2},[9]$. 


\section{$\begin{array}{llll}\text { J.Thi-Qar Sci. } & \text { No. (2) } & \text { Vol.1 } & \text { Aug./2008 }\end{array}$}

\section{Proposition 4.6:}

Let $S$ be left regular,then every intuitionistic fuzzy left ideal of $S$ is intuitionistic fuzzy semiprime.

\section{Proof:}

Let $A=\left(\mu_{A}, \gamma_{A}\right)$ be an intuitionistic fuzzy left ideal of $S$ and let $a \in S$, then there exists an element $\mathrm{x}$ in $\mathrm{S}$ such that $a=x a^{2}$, then $\mu_{A}(a)=\mu_{A}\left(x a^{2}\right) \geq \mu_{A}\left(a^{2}\right)$ since $\mathrm{S}$ is left regular.

Also,we have $\gamma_{A}(a)=\gamma_{A}\left(x a^{2}\right) \leq \gamma_{A}\left(a^{2}\right)$; that is A is fuzzy semiprime.

\section{Proposition 4.7:}

Let $A=\left(\mu_{A}, \gamma_{A}\right)$ be an intuitionistic fuzzy ideal of $\mathrm{S}$.If $\mathrm{S}$ is intra-regular,then $A=\left(\mu_{A}, \gamma_{A}\right)$ is intuitionistic fuzzy semiprime.

\section{Proof:}

Let $\mathrm{a} \in S$. Then there exist $\mathrm{x}, \mathrm{y} \in S$ such that $\mathrm{a}=\mathrm{x} a^{2} y$, thus $\mu_{A}(a)=\mu_{A}\left(x a^{2} y\right) \geq \mu_{A}\left(x a^{2} y\right) \geq \mu_{A}\left(a^{2} y\right) \geq \mu_{A}\left(a^{2}\right)$

and $\gamma_{A}(a)=\gamma_{A}\left(x a^{2} y\right) \leq \gamma_{A}\left(a^{2} y\right) \leq \gamma_{A}\left(a^{2}\right)$

Hence, $A=\left(\mu_{A}, \gamma_{A}\right)$ is intuitionistic fuzzy semiprime.

\section{Definition 4.8[2]:}

An intuitionistic fuzzy subsemigroup $A=\left(\mu_{A}, \gamma_{A}\right)$ of $\mathrm{S}$ is called an intuitionistic fuzzy interior ideal of $\mathrm{S}$ if

1. $\mu_{A}(x a y) \geq \mu_{A}(a)$

2. $\gamma_{A}(x a y) \leq \gamma_{A}(a)$, for all $\mathrm{x}, \mathrm{y}, \mathrm{a} \in S$.

\section{Proposition 4.9:}

Let $A=\left(\mu_{A}, \gamma_{A}\right)$ be an intuitionistic fuzzy interior ideal of $\mathrm{S}$.If $\mathrm{S}$ is an intraregular,then $A=\left(\mu_{A}, \gamma_{A}\right)$ is intuitionistic fuzzy semiprime.

\section{Proof:}

It follows by definition 4.4 and definition 4.8 . 


\title{
J.Thi-Qar Sci. No. (2) Vol.1 Aug./2008
}

\section{REFRENCES :}

[1] Zadeh.L.A.,(1965),"Fuzzy Sets", Information and Control,8:338-353.

[2] Goguen J.A.,(1967),"L-Fuzzy Sets",Math.Anal.Appl.,18:145-174.

[3] Atanassov K.T.,(1986),"Intuitionistic fuzzy sets",Fuzzy sets and Systems,20:87-96.

[4] Lajos S.,(1961),"Generalized ideals in semigroup',Scta Sci.Math.,22:217-222.

[5] Kuroki N.,(1982),"Fuzzy semiprime ideals in semigroups",Fuzzy sets and Systems, 8:71-79.

[6] Martines L. ,(1996),"Fuzzy modules over fuzzy rings in connection with Fuzzy ideal of Fuzzy ring ". J.Fuzzy Mathematics , 4:843-857.

[7] Roh,E.H and Lee,J.G.(2006),"intutionstic Q-fuzy subalgebras of BCK/BCI-algebras",international Mathemtical Forum,24:1167-1174.

[8] Lajos S.,(1997),"(1,2)-ideal characterizations of unions of groups",Kobe J.Math. 5:447-450.

[9] Kuroki N.,(1981),N."On fuzzy ideals and fuzzy bi-ideals in semigroups",Fuzzy sets and Systems,5:203215.

[10] Atanassov K.T.,(1994),"New operations defined over the Intuitionistic fuzzy sets", Fuzzy sets and Systems ,61:137-142.

\section{Some types of fuzzy ideals in semigroups}

\author{
ريبيع هادي جاري اكرم برزان عطار \\ قسم الرياضيات- كلية التربية- جامعة ذي قار \\ الملخص \\ درسنا في هذا البحث مفهوم المثالي الضبابي في شبه الزمرة وأعطينـا بعض الخواص وذكرنـا بعض

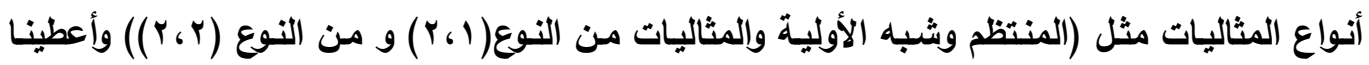 \\ بعض العلاقات بينهما.
}

